\title{
Soldiers' Perspectives of Insomnia Behavioral Treatment in a Primary Care Setting
}

\section{Juliana S Ee ${ }^{1}$, Cristóbal S Berry-Cabán ${ }^{2 *}$, Dana R Nguyen ${ }^{3}$, Madina Boyd ${ }^{1}$, Nick Bennett ${ }^{4}$, Thomas Beltran ${ }^{2}$ and Michele Williams ${ }^{2}$}

\author{
${ }^{1}$ Department of Family Medicine, Womack Army Medical Center, NC, USA \\ ${ }^{2}$ Department of Clinical Investigation, Womack Army Medical Center, NC, USA \\ ${ }^{3}$ Department of Family Medicine, Uniformed Services University, MD, USA \\ ${ }^{4}$ Farrelly Health Clinic, KS, USA
}

*Corresponding author: Cristóbal S Berry Cabán, Department of Clinical Investigation, Womack Army Medical Center, Fort Bragg, NC, USA, E-mail: cristobal.s.berry-caban.civ@mail.mil

\begin{abstract}
Introduction: Insomnia is a highly prevalent sleep disorder in the US. Among the military population, $41 \%$ of active duty service members reported less than 5 hours sleep per night, and there was nearly a 20 -fold increase in incidence of insomnia among service members between 2000 and 2009. Given that most soldiers with insomnia seek initial treatment in the primary care setting, an understanding of soldiers' treatment expectations and preferences may contribute to treatment success. This study examines soldiers' perspectives on behavioral insomnia treatment in the primary care setting.
\end{abstract}

Methods: This was a cross-sectional survey study conducted at two outpatient clinic waiting areas in a military treatment facility. The survey assessed soldiers' experience of insomnia, treatment expectations and preferences, and willingness to practice behavioral techniques. Participation was voluntary and responses were anonymous.

Results: Two hundred active duty service members (177 males: mean age $=29$ years) completed the survey. More than $40 \%$ of the respondents reported trouble falling or staying asleep on most days during a typical week; the average sleep time reported was 5.7 hours $(\mathrm{SD}=1.4$ ) during a 24-hour period over the previous 30 days. More than $80 \%$ of the respondents indicated interest in learning behavioral skills to sleep better, in being taught good sleep habits, or in getting assistance with developing and sticking to a behavioral treatment plan. Willingness to practice behavioral techniques was moderately strong. Fifty-nine percent of the respondents indicated preference for behavioral treatment only or behavioral first and then medications.

Discussion: Cognitive-behavioral treatment for insomnia is an alternative to short-term sedative-hypnotic treatment. Soldiers' interest and willingness to practice behavioral sleep techniques are strong. Primary care providers should move beyond providing sleep hygiene by adding education on ways to strengthen the bed and sleep association and to establish a consistent and regular sleep-wake schedule.

\section{Keywords}

Insomnia, Sleep, Soldiers, Military, Cognitive-behavioral, Primary care

\section{Introduction}

Unaddressed sleep disorders such as insomnia have been recognized as having potentially serious consequences on an individual's functioning and overall quality of life, leading to increased risk of developing hypertension, obesity, and cardiac disease [1-4]. Insomnia can exist as a primary disorder or comorbid with other psychiatric conditions such as depression, anxiety, and other psychiatrically related impairments $[5,6]$. Thus, sleep disruption can be a major contributor to the development of a significant psychiatric condition that is considered a leading cause of disability among men and women in the United States and one of the 10 leading disorders for global disease [6].

Insomnia is a highly prevalent sleep disorder in the US. Around $30 \%$ of the adult population reported intermittent symptoms of insomnia, while $5 \%$ to $10 \%$ have been identified as having insomnia specific disorders $[7,8]$. The National Sleep Foundation's annual Sleep in America survey found that two-thirds of Americans feel their sleep needs are not being met during the week, and that they are searching for ways to cope [7-9]. Among the military population, $41 \%$ of active duty service members reported an average of less than 5 hours sleep per night, and there has been a nearly 20 -fold increase in incidence of insomnia among service members between 2000 and 2009 [10,11].

When compared to their civilian counterparts, military personnel face unique sleep-architecture altering experiences and are often subject to continuous sleep disruption due to mission requirements, combat-simulation exercises such as night missions and early morning wake-up calls, deployments, unit activities at remote geographical locations, and frequent separations from the family [12]. Furthermore, the rapid operational tempo and varying degrees of combat exposure, as well as continuous shifts in the demands of multiple missions have been linked to difficulties with sleep onset latency and sleep duration $[12,13]$.

Regular sleep disruptions were reported by active duty service members as concentration difficulty, which was reflected in decreased marksmanship scores, diminished physical abilities, and reduced 
overall fitness performance [14,15]. Finally, poor sleep quality identified in soldiers prior to deployment has been associated with an increased risk of developing subsequent psychiatric difficulties and an increase in risky health behaviors post-deployment, thus potentially highlighting sleep complaints as an early indicator of vulnerability $[13,15]$.

Continuous sleep deprivation and poor sleep practices prior to deployment have been linked as positive screens for new-onset posttraumatic stress disorder, depression, and other anxiety related disorders; hence they have been identified as potential risk factors in the active duty personnel for experiencing impaired functioning post-deployment [16].

Sleep problems have also been associated with mild traumatic brain injury (mTBI) and the development of mental health symptoms after returning from deployments. According to Macera, et al. military personnel who experienced mTBI complained of sleep difficulties immediately after their return from deployment and endorsed psychiatric difficulties, to include posttraumatic stress disorder and major depressive disorder several months later [15].

These data further strengthens evidence that identifying sleep disruption early in the process can be a preventative measure that might aid in addressing the latent onset of psychiatric conditions in military personnel [12]. Finally, identified but potentially overlooked, insomnia has been noted as a risk factor in suicidal ideation and suicide attempts in the military $[12,17]$. Lentino, et al. identified that "emotional health as measured by the Army GAT [Global Assessment Tool], was highly dependent on sleep quality, with poor sleepers being 23 times more likely to have scored in the lowest quartile for emotional health" [14].

Insufficient sleep and its impact on individual performance, adverse physical consequences, and potential increase in mortality in the military and population at large has increasingly become a focus of research [14]. Notably, development of more effective, evidencebased interventions, including the use of both medication and behaviorally-based treatments, have been a particular area of interest in evaluating current trends for making first contact with any given provider.

The importance of understanding the nature of sleep disorders and addressing their underlying symptoms for military personnel operating in high-risk environments and technically demanding occupations cannot be over-emphasized [18]. The level of impairment as well as subjective and objective perception of health-related decline in quality of life have been well-documented as leading to increased emergency room visits, laboratory testing requests, and overall increase in health care utilization when compared to patients with no insomnia [19].

Insomnia is one of the most common conditions presented in primary care setting, with patient estimates ranging from $10 \%$ to $50 \%$ [20]. Most patients with insomnia seek initial treatment in a primary care setting and discuss their concerns with their primary care provider (PCP); thus, given the prevalence of insomnia and patients' potential desire to obtain sleep aids or any other assistance, most PCP can expect to encounter these patients in their practice [21]. Furthermore, the military population is more likely to seek sleep-related treatment through their PCP due to potential stigma associated with approaching a behavioral healthcare provider [22].

Most PCP typically treat the problem by recommending sleep hygiene practices and prescribing medications [23]. The pharmacologic therapy includes short- to intermediate-acting benzodiazepine hypnotics, non-benzodiazepine hypnotics, or sedating low-dose antidepressants. These medications, however, present their own risks, including excessive drowsiness, and physical and psychological dependence with long-term use [23].

Notably, for the military population the ability to receive and remain on medication for the long term might present specific hosts of issues depending on the individual's occupational specialty and current duties (e.g., flight status, airborne status, deployment, specialty schools, etc.) thus, more conservative approaches as a firstline treatment of choice are recommended.

Sleep experts agree that short-term sedative-hypnotic treatment should be supplemented with psychological and behavioral therapies whenever possible [23]. The effectiveness of cognitive-behavioral treatment (CBT-I) has been well-documented and recommended as standard first-line treatment for insomnia [23-30]. Furthermore, behavioral approaches appear to be a preferred treatment for insomnia patients suffering with comorbid conditions such as chronic pain and heart failure, due to longer efficacy and absence of side-effects when compared to pharmacological interventions [24]. Additionally, behavioral interventions have produced reductions in sleep-related symptoms for up to $73 \%$ of insomnia patients referred for treatment, and they appear to have much longer lasting efficacy following treatment termination $[25,27]$.

Cognitive-behavioral interventions for insomnia (CBT-I) comprised four core components that include: sleep hygiene practices; stimulus control; sleep restriction; and stress management and cognitive techniques to reduce sleep-interfering arousal and to prepare the mind and body for sleep. CBT-I has been found to be effective in as little as a single session group treatment [31].

Despite its effectiveness, CBT-I has been under-utilized by primary care providers due in part to perceived or real barriers that include time efficiency, physicians' perceptions of patients' expectations, patients' acceptance of the treatment modality, and the skills experience of the primary care practitioners [32]. Most clinical encounters involve a dyadic interaction between the physician and the patient. The providers' role is to bring to the encounter their respective expert knowledge, beliefs, preferences, and expectations about how best to treat a condition. Clinicians' understanding of patients' attitudes and beliefs about insomnia and its treatment is perceived to be closely related to the successful outcome of therapeutic approach. Attempts have been made to investigate treatment preference and satisfaction with group treatment in the individual with chronic insomnia, and comparison of acceptance for psychological and pharmacological therapies for insomnia have been conducted among those in treatment at specialty sleep clinics [33-37].

The purpose of this exploratory study was to survey active duty service members' perspectives and preferences on behavioral insomnia treatment in the primary care setting. Given that most soldiers with insomnia seek initial treatment in the primary care setting, an understanding of soldiers' expectations and preferences regarding treatment may contribute to treatment success. The information can also be used to shape training curriculum for physician residents.

\section{Methods}

This was a cross-sectional survey study performed at the Womack Army Medical Center (WAMC) outpatient clinics. A survey instrument was assembled, using information from relevant insomnia treatment studies. The final survey contained items that assessed respondents' self-reported total hours of sleep in a 24-hour period over the past 30 days; their experience of trouble falling asleep and trouble staying asleep; their perception of their health status; their expectations about what interventions they want their primary care physicians to provide; their willingness to practice a series of behavioral techniques that have been established in the sleep literature as the "standard" for insomnia treatment; and their treatment preferences (e.g., medications only, behavioral only, medications then behavioral, behavioral then medications, and concurrent combination of medications and behavioral). Respondents also provided socio-demographic data including gender, age, marital status, race/ethnicity, highest educational level, and number of deployments.

Two hundred responses to the survey were collected via convenience sampling from active duty soldiers in the waiting areas 
of the Physical Examination and Family Medicine outpatient clinics. A power analysis was conducted to estimate the number of survey respondents required for examination of mean differences. That analysis indicated that 128 respondents were required for an $80 \%$ chance of rejecting null hypotheses (two-tailed, one-way ANOVA) at the alpha level of 0.05 . Since the sample was drawn from an army medical center, it was anticipated that the sample would be composed of approximately $80 \%$ male respondents. We inflated the number surveyed for a more adequate sampling of female respondents.

Participation in the study was voluntary. Respondents' information, including names that could link an individual to the data was not recorded to assure confidentiality and anonymity. All active duty soldiers above 18 years of age were included. Active duty soldiers who declined to participate and civilian patients were excluded. The survey objectives were explained and an informed verbal consent was obtained prior to distribution of the survey. The survey took approximately 10-15 minutes to complete. This project was approved by the WAMC Institutional Review Board.

Statistical analyses were conducted using IBM SPSS Statistics 22 (IBM Corporation, Armonk, NY). Descriptive statistics including frequencies, means, standard deviations (SD), and range were calculated, and, where appropriate, chi-square and ANOVA were used to examine group differences.

\section{Results}

A total of 200 active duty soldiers completed the survey, $88.5 \%$ were males $(n=177)$; the mean age was 29 years $(S D=7.03)$. Ethnic or racial identity reported by the respondents was as follows: white, non-Hispanic white $61 \%$ non-Hispanic blacks $18.5 \%$, Hispanics $13 \%$, and Asian/Pacific Islander or other 7.5\%.

Respondents were asked to assess their perceived health status using a 5 point Likert-type scale with ratings of poor, fair, good, very good, and excellent. Two individuals (1\%) reported being in poor health; 18 individuals (9\%) reported fair health; 73 individuals (36.5\%) reported good health; 81 individuals (40.5\%) reported very good health, and 26 individuals (13\%) reported excellent health. No group differences were found for perceived health status based on gender, race/ethnicity, marital status, level of education, or number of deployments $(P>0.05)$. A Kruskal-Wallis test compared respondents' treatment preferences with their self-evaluated health status showed no relationship $(P=0.62)$.

Overall, respondents reported an average total sleep time of 5.7 hours during a 24 -hour period over the previous 30 days $(\mathrm{SD}=1.4$; range $=2.0-11.0$ hours). Forty percent of the respondents reported trouble falling asleep 'more than half the week' during a typical week in the past 30 days while $46 \%$ reported trouble staying asleep. A oneway ANOVA was conducted to examine differences in the amount of sleep individuals' reported based on gender and race. A significant difference was found between males and females (mean $=5.7$ vs. 6.4 hours; $P=0.04$ ) which indicated that females reported getting more sleep than males. However, results indicated there was no difference in the number of hours slept based on reported race/ethnicity ( $P$ $=0.47)$, level of education $(P=0.13)$, marital status $(P=0.70)$, or number of deployments $(P=0.44)$.

No differences were found between individuals' preferred treatment for sleep problems based on the frequency of their experience having trouble falling asleep or trouble staying asleep $(P>0.05)$. Additionally, there was no difference in treatment preference and number of hours respondents were willing to devote to treatment $(P=0.30)$ or between treatment preference and number of deployments $(P=0.71)$.

Respondents' treatment expectations were assessed via the statement: "If I see my doctor for treatment of my sleep problems, I would like my doctor to...". A list of options was provided and respondents selected responses, using a 6-point Likert scale, which ranged from "strongly disagree" to "strongly agree". Eighty-five percent of the respondents "slightly agree" to "strongly agree" that
Table 1: Treatment expectations (proportion reporting "slightly agree" to "strongly agree"; $n=200$ ).

\section{Expectations}

Find out why I have sleep problems

Percent

Teach me behavioral skills to sleep better

Teach me good sleep habits

$85 \%$

Help me develop a behavior plan to treat my sleep problem

Ask about my stress

$83 \%$

$82 \%$

$82 \%$

Ask about my progress in sticking to the behavioral plan $80 \%$

Help me find out what difficulties interfere with sticking to the plan

Coach me on behavioral skills until I get them right

Prescribe sleep medication

$80 \%$

$78 \%$

$57 \%$

Table 2: Willingness to practice behavioral techniques (proportion of respondents rating their willingness "frequently" or "usually"; $n=200$ )

\begin{tabular}{|l|l|}
\hline Techniques & Percent \\
\hline Make bedroom comfortable for sleep & $78 \%$ \\
\hline Exercise regularly & $66 \%$ \\
\hline Avoid alcohol before bedtime & $66 \%$ \\
\hline Avoid nicotine before bedtime & $62 \%$ \\
\hline Avoid caffeine before bedtime & $61 \%$ \\
\hline Use the bed only for sleep (and sex) & $58 \%$ \\
\hline Stop watching TV in bed while waiting to fall asleep & $57 \%$ \\
\hline Stay on a regular sleep-wake schedule & $51 \%$ \\
\hline Avoid naps during the daytime & $48 \%$ \\
\hline Get out of bed when unable to fall asleep within 15-20 minutes & $38 \%$ \\
\hline Practice relaxation before bedtime & $37 \%$ \\
\hline Go to another room and not return to bed until feeling sleepy & $33 \%$ \\
\hline
\end{tabular}

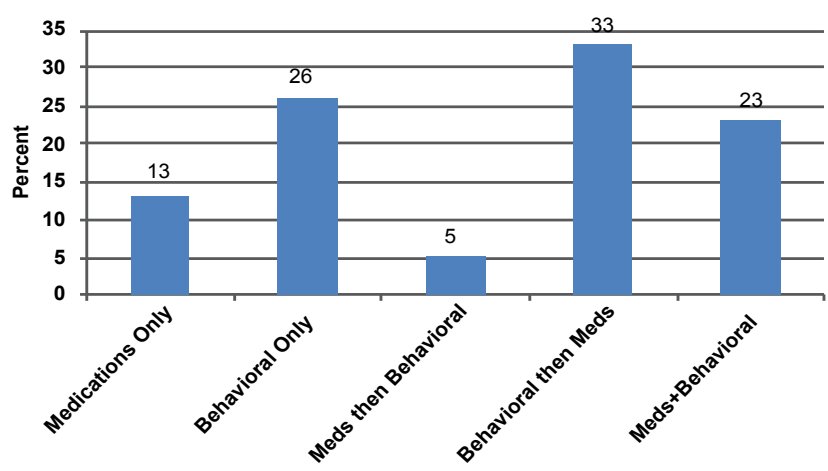

Figure 1: Treatment preferences.

they want their healthcare provider to "find out why I have a sleep problem"; $85 \%$ "slightly agree" to "strongly agree" that they want their healthcare provider to "teach me behavioral skills to sleep better"; $83 \%$ "teach me good sleep habits"; $82 \%$ "ask about my stress"; $80 \%$ "ask about my progress in sticking to the behavioral plan"; $80 \%$ "help me find out what difficulties interfere with sticking to the plan"; $78 \%$ "coach me on behavioral skills until I get them right"; and 57\% "prescribe sleep medication" (Table 1). Respondents' self-rated willingness to practice behavioral techniques is summarized in table 2 .

Respondents' preferences for treatment approaches were as follows: $13 \%(\mathrm{n}=26)$ indicated preferences for medications only; $26 \%(\mathrm{n}=52)$ behavioral methods only; $5 \%(\mathrm{n}=10)$ medications first, then behavioral; 33\% $(n=66)$ behavioral first, then medications; and $23 \%(n=46)$ selected a concurrent combination of medications and behavioral methods (Figure 1).

\section{Discussion}

This aim of this exploratory study was to investigate the treatment preferences of active duty service members consulting their primary care providers for insomnia, in particular, their expectations, willingness to practice behavioral techniques, and preferences for insomnia treatments in the primary care setting. The importance of patients' involvement in their healthcare is increasingly recognized by the medical profession. For patients to be involved, their priorities, 
expectations, and preferences must be identified and addressed. A favorable acceptance of behavioral treatments will provide data-driven support for the development of an insomnia behavioral treatment curriculum for physician residents in training and for expanding the insomnia treatment tool box of primary care providers.

Our study demonstrates that soldiers have a strong interest in learning behavioral treatments for their sleep difficulties. Up to 85\% of the respondents expect their physician to teach them behavioral skills to sleep better or to assist them in developing a behavioral treatment plan for their sleep problems. Behavioral treatment first and then medications was selected by $33 \%$ of the respondents whereas medications first and then behavioral treatment was preferred by only $5 \%$ of the respondents. About $23 \%$ of the respondents indicated a preference for a concurrent combination of both pharmacologic and behavioral treatments.

Cognitive-behavioral interventions for sleep comprised four essential components: sleep hygiene, stimulus control (strengthening the association between bed and sleep), sleep restriction, and training the mind and body for sleep through muscle relaxation, articulatory suppression, and cognitive control. Less than $40 \%$ of the respondents indicated that they were "frequently" or "usually" willing to practice techniques that strengthen the association between the bed and sleep (e.g., getting out of bed when unable to fall asleep, going to another room and not return to bed until feeling sleepy). Clearly, more attention must be devoted to explaining the rationale for engaging in actions that strengthen the link between bed and sleep. Additionally, more attention must also be directed toward explaining the rationale for staying on a regular and consistent sleep-wake schedule as only $51 \%$ of the respondents indicated their willingness to practice this technique "frequently" or "usually."

Given the strong preference for behavioral treatments, more interest should be directed toward providing behavioral treatments in the primary care setting. Edinger and Sampson have demonstrated successful outcomes using an abbreviated 2-session CBT model for insomnia in the primary care setting [38]. Researchers have demonstrated favorable outcomes for a one-session CBT-I delivered in a group format [31]. Thus, CBT-I can be effectively and efficiently provided in the primary care setting.

This study has several limitations. First, this was a cross-sectional survey study that utilized a convenience sample of active duty service members in two outpatient clinic waiting areas in a military treatment facility. Second, the survey instrument, though constructed from the sleep studies literature, is currently not validated, given the exploratory nature of the study. Third, the findings relied on selfreport and no attempts were made to clinically diagnose insomnia disorder or to examine co-morbid conditions that may have an impact on respondents' treatment expectations or preferences. These limitations diminished the generalizability of the study findings. Future studies should consider surveying a wide range of active duty service members in other settings as well as non-military population, collecting data from primary care providers regarding patients' selection of insomnia treatment modalities, and examining comorbid conditions that may influence expectations and preferences.

Despite these limitations, the study findings showed that interest in and willingness to practice behavioral treatment for insomnia is strong among soldiers in the primary care setting. Coaching patients in cognitive-behavioral skills to improve sleep should be considered beyond merely providing sleep hygiene education. A focused insomnia behavioral treatment curriculum could be developed and training provided to physician residents and primary care providers to expand their insomnia treatment toolbox.

\section{Acknowledgement}

Dr. Ee wishes to thank Dr. Colleen Fogarty, University of Rochester Medical Center and Dr. Michael Floyd, East Tennessee State University for their mentoring during her participation in the Society of Teachers of Family Medicine, Behavioral Science/Family Systems Educator Fellowship. This project was completed as part of the fellowship learning activities. The views expressed herein are those of the authors and do not reflect the official policy of the Department of the Army, Department of Defense, or the US Government.

\section{References}

1. Chang P, Ford D, Mead L, Cooper-Patrick L, Klag M (1997) Insomnia in young men and subsequent depression: The John Hopkins Precursors Study. Am J Epidemiol 146: 105-114.

2. Vgontzas AN, Liao D, Bixler EO, Chrousos GP, Vela-Bueno A (2009) Insomnia with objective short sleep duration is associated with a high risk for hypertension. Sleep 32: 491-497.

3. Zammit G, Weiner J, Damato N, Sillup G, McMillan C (1999) Quality of life in people with insomnia. Sleep 22: S379-385.

4. Bonnet $\mathrm{MH}$, Arand DL (2007) Cardiovascular implications of poor sleep. Sleep Medicine Clinics 2: 529-538.

5. Mitchell MD, Gehrman P, Perlis M, Umscheid CA (2012) Comparative effectiveness of cognitive behavioral therapy for insomnia: a systematic review. BMC Family Practice 13: 40.

6. Baglioni C, Battagliese G, Feige B, Spiegelhalder K, Nissen C, et al. (2011) Insomnia as a predictor of depression: a meta-analytic evaluation of longitudinal epidemiological studies. J Affect Disord 135: 10-19.

7. National Sleep Foundation (2010) 2010 Sleep in America ${ }^{\circledR}$ Poll.

8. National Sleep Foundation (2011) 2011 Sleep in America ${ }^{\circledR}$ Poll.

9. Brems C (2015) A yoga stress reduction intervention for university faculty, staff, and graduate students. Int J Yoga Therap 25: 61-77.

10. Mysliwiec V, Gill J, Lee H, Baxter T, Pierce R, et al. (2013) Sleep disorders in US military personnel: a high rate of comorbid insomnia and obstructive sleep apnea. Chest 144: 549-557.

11. (2010) Armed Forces Health Surveillance Center. Insomnia, active component, U.S. Armed Forces, January 2000-December 2009. Medical Surveillance Monthy Report 17: 12-15.

12. Lande RG, Gragnani C (2013) Sleep trends of active-duty service members referred for psychiatric care: a descriptive study. J Am Osteopathic Assoc 113: $144-150$.

13. Swinkels CM, Ulmer CS, Beckham JC, Buse N, Calhoun PS (2013) The association of sleep duration, mental health, and health risk behaviors among US Afghanistan/Iraq era veterans. Sleep 36: 1019-1025.

14. Lentino CV, Purvis DL, Murphy KJ, Deuster PA (2013) Sleep as a component of the performance triad: the importance of sleep in a military population. US Army Med Dep J 98-108.

15. Macera CA, Aralis HJ, Rauh MJ, MacGregor AJ (2013) Do sleep problems mediate the relationship between traumatic brain injury and development of mental health symptoms after deployment? Sleep 36: 83-90.

16. Gehrman P, Seelig AD, Jacobson IG, Boyko EJ, Hooper TI, et al. (2013) Predeployment sleep duration and insomnia symptoms as risk factors for new-onset mental health disorders following military deployment. Sleep 36: 1009-1018.

17. Ribeiro JD, Pease JL, Gutierrez PM, Silva C, Bernert RA, et al. (2012) Sleep problems outperform depression and hopelessness as cross-sectional and longitudinal predictors of suicidal ideation and behavior in young adults in the military. J Affect Disord 136: 743-750.

18. Mysliwiec V, McGraw L, Pierce R, Smith P, Trapp B, et al. (2013) Sleep disorders and associated medical comorbidities in active duty military personnel. Sleep 36: 167-174.

19. Roth T, Franklin M, Bramley TJ (2007) The state of insomnia and emerging trends. Am J Manag Care 13: S117-120.

20. Arroll B, Fernando A, Falloon K, Goodyear-Smith F, Samaranayake C, et al. (2012) Prevalence of causes of insomnia in primary care: a cross-sectional study. Br J Gen Pract 62: e99-103.

21. Simon GE, VonKorff M (1997) Prevalence, burden, and treatment of insomnia in primary care. Am J Psychiatry 154: 1417-1423.

22. Morin CM, LeBlanc M, Daley M, Gregoire J, Merette C (2006) Epidemiology of insomnia: prevalence, self-help treatments, consultations, and determinants of help-seeking behaviors. Sleep Med 7: 123-130.

23. Schutte-Rodin S, Broch L, Buysse D, Dorsey C, Sateia M (2008) Clinica guideline for the evaluation and management of chronic insomnia in adults. $J$ Clin Sleep Med 4: 487-504

24. Andrews LK, Coviello J, Hurley E, Rose L, Redeker NS (2013) "I'd eat bucket of nails if you told me it would help me sleep:" Perceptions of insomnia and its treatment in patients with stable heart failure. Heart Lung 42: 339-345.

25. Benca RM (2005) Diagnosis and treatment of chronic insomnia: a review. Psychiatr Serv 56: 332-343. 
26. Brooks AT, Wallen GR (2014) Sleep disturbances in individuals with alcoholrelated disorders: a review of cognitive-behavioral therapy for insomnia (CBT-I) and associated non-pharmacological therapies. Subst Abuse 8: 5562.

27. Chambers MJ, Alexander SD (1992) Assessment and prediction of outcome for a brief behavioral insomnia treatment program. J Behav Ther Exp Psychiatry 23: 289-297.

28. Edinger JD, Wohlgemuth WK, Radtke RA, Marsh GR, Quillian RE (2001) Cognitive behavioral therapy for treatment of chronic primary insomnia. JAMA 285: 1856-1864.

29. Lichstein K, Wilson N, Johnson C (2000) Psychological treatment of secondary insomnia. Psychol Aging 15: 232-240.

30. Morin C, Colecchi C, Stone J, Sood R, Brink D (1999) Behavioral and pharmacological therapies for late-life insomnia: A randomized controlled trial. JAMA 281: 991-999.

31. Brooks M (2013) Single-session group CBT effective for insomnia. Medscape.

32. Cockburn J, Pit S (1997) Prescribing behaviour in clinical practice: patients expectations and doctors' perceptions of patients' expectations - a questionnaire study. BMJ 315: 520-523.
33. Vincent N, Lewycky S, Finnegan $\mathrm{H}$ (2008) Barriers to engagement in sleep restriction and stimulus control in chronic insomnia. J Consult Clin Psychol 76: $820-828$

34. Morin CM, Gaulier B, Barry T, Kowatch RA (1992) Patients' acceptance of psychological and pharmacological therapies for insomnia. Sleep 15: 302 305.

35. Jacobs GD, Pace-Schott EF, Stickgold R, Otto MW (2004) Cognitive behavior therapy and pharmacotherapy for insomnia: a randomized controlled trial and direct comparison. Arch Intern Med 164: 1888-1896.

36. Sivertsen B, Omvik S, Pallesen S, Bjorvatn B, Havik OE, et al. (2006) Cognitive behavioral therapy vs zopiclone for treatment of chronic primary insomnia in older adults: a randomized controlled trial. JAMA 295: 2851-2858.

37. Morin CM, Vallières A, Guay B, Ivers H, Savard J, et al. (2009) Cognitive behavioral therapy, singly and combined with medication, for persistent insomnia: a randomized controlled trial. JAMA 301: 2005-2015.

38. Edinger JD, Sampson W (2003) A primary care "friendly" cognitive behavioral insomnia therapy. Sleep 26: 177-182. 